\title{
Molecules in motion: Michael Sheetz, James Spudich, and Ronald Vale receive the 2012 Albert Lasker Basic Medical Research Award
}

\begin{abstract}
$T_{h}$
he Albert and Mary Lasker Foundation honors three pioneers in the field of molecular motor proteins, Michael Sheetz, James Spudich, and Ronald Vale (Figure 1), for their contribution to uncovering how these proteins catalyze movement. Sheetz and Spudich developed the first biochemical assay to reconstitute myosin motor activity and showed that myosin and ATP alone were sufficient to direct transport along actin filaments. Fueled by this discovery, Vale and Sheetz examined transport in giant squid axon extracts and discovered a new motor, kinesin, that moves along microtubules. Cumulatively, their work opened the door for understanding how motor proteins drive transport of a host of molecules involved in numerous cellular processes, including cell polarity, cell division, cellular movement, and signal transduction.
\end{abstract}

\section{Muscle movement}

Much of the early research on cellular movement focused on understanding muscle contraction. Filaments of actin and myosin form a banding pattern in muscle that is readily visible by light microscopy, with a characteristic striated appearance. The functional unit of muscle, termed a sarcomere, repeats at regular intervals along the muscle fibers. Measurements from contracted, resting, and stretched muscle using high-resolution light microscopy supported the notion that contraction was due to the sliding of actin and myosin filaments $(1,2)$. In 1969, preeminent British scientist Hugh Huxley proposed that crossbridges that connect actin and myosin generate the sliding motion that moves actin during muscle contraction (3). His model, termed the swinging crossbridge hypothesis, predicted that these crossbridges bind and release in a cyclical manner to produce the force that causes actin sliding, thus propelling movement and muscle contraction. While an elegant model, it was difficult to test and remained controversial for many years. Soon after the swinging crossbridge hypothesis was proposed,
James Spudich joined the Huxley laboratory as a young postdoctoral researcher. From his experiences there, Spudich was keenly aware of the need for a biochemical assay for the function of interest, movement, and to explore the mechanism of energy transduction by the myosin protein. In a recent interview with the JCI, Spudich recalled that many of the biophysical experiments from the 1970s did not support the swinging crossbridge model, "Frankly, people were beginning to think that it may not work the way Hugh had proposed." When Spudich branched off and opened his own research laboratory, he set out to rigorously and definitively test models of energy transduction by myosin. In addition, he developed a strong interest in the nascent field of nonmuscle myosins.

\section{Recapitulating motion in the laboratory}

First at the University of California, San Francisco, and later at Stanford, a primary goal in Spudich's laboratory was to develop an in vitro assay using only purified components to examine myosin motility along actin. He and his colleagues took a multipronged approach, using biophysical, biochemical, and genetic techniques to uncover the mechanism of myosin action. Along with postdoctoral fellow Margaret Clarke, Spudich identified a model organism for studying nonmuscle actin and myosin biochemistry, the amoeba Dictyostelium (4). Early studies with the organism involved actin filaments attached to tiny polystyrene beads, which provided encouragement for an in vitro motility assay. Years later, the Spudich laboratory remarkably developed genetic tools to examine Dictyostelium cells lacking myosin and to test the ability of various myosin mutants to compensate. Spudich recalled, "My graduate student Arturo De Lozanne unexpectedly discovered that one could use the genetic approach of homologous recombination with Dictyostelium. That did a lot for us because we were then able to create a cell that did not contain myosin. That cell was unable to divide into two daughter cells (5). Then, we made mutations in the myosin and put those mutated myosins back into the cell that lacked the myosin to follow the

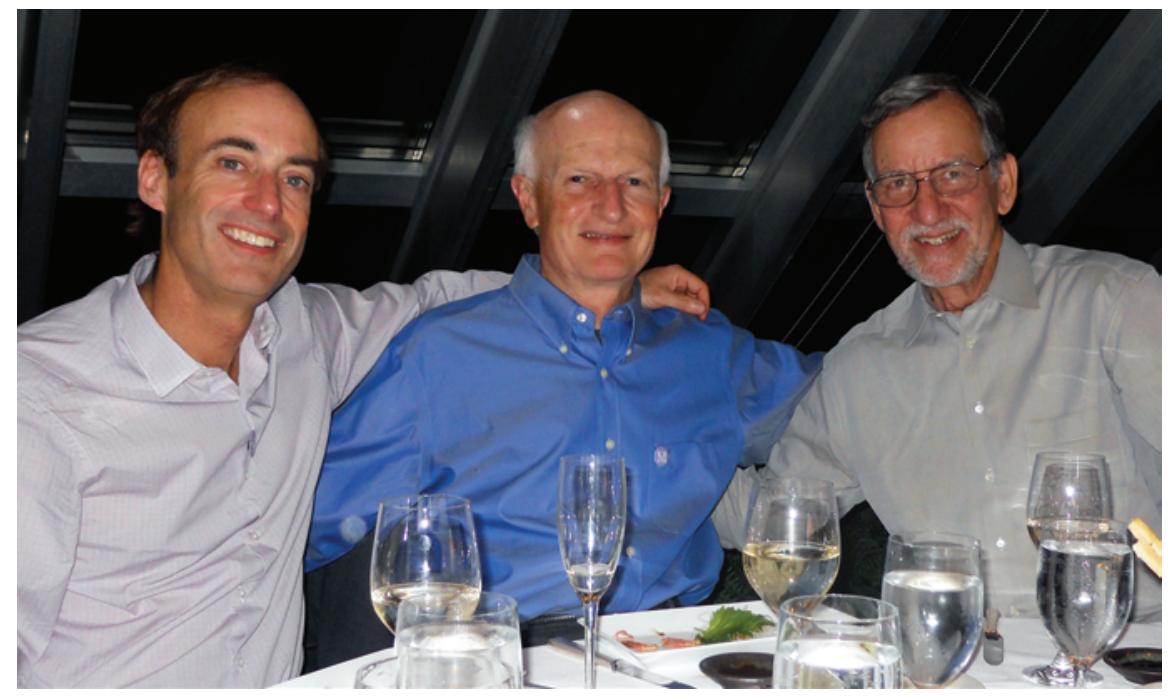

Figure 1

Ronald Vale (left), Michael Sheetz (center), and James Spudich (right) are the winners of the 2012 Albert Lasker Basic Medical Research Award. 


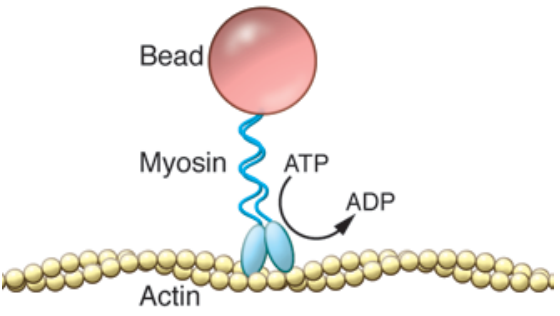

Figure 2

An in vitro assay to test myosin motility. Michael Sheetz and James Spudich developed the first biochemical assay to examine the movement of myosin along actin filaments. Using myosin bound to fluorescently labeled beads, they tracked myosin movement microscopically along actin-aligned filaments, first in the alga Nitella (6) and later using purified actin bound to glass slides (7). Spudich and colleagues later showed that the S1 head of myosin both binds to actin and hydrolyzes ATP to generate motion (14).

behavior in vivo that resulted from those different mutations." Despite much progress, several technical hurdles left the Spudich laboratory still searching for an assay to reconstitute myosin movement along actin with known, purified components.

A major breakthrough in the development of an in vitro assay for myosin movement along actin occurred when Michael Sheetz joined the Spudich laboratory in 1982. Spudich recalled, "Our model was that actin and myosin would move without anything else, but there was no proof that that was the way nature worked. Minimally, it could have easily required another component besides pure actin and myosin. So when it wasn't working, it could have been the alignment of the actin filaments, it could have been that the myosin on the beads wasn't functional, or it could have been that nature didn't really work this way and we were testing a model that would never work. We strongly suspected, however, that what was wrong in our previous experiments was that we just didn't have a well-aligned lawn of actin filaments to put myosin-coated beads on." In a new approach, Sheetz and Spudich took advantage of well-aligned actin filaments in a species of a giant alga to examine the motion of purified myosin conjugated to fluorescently labeled beads (Figure 2 and ref. 6). Sheetz told the JCI, "We both recalled that the large alga Nitella, which has single cells that are $3 \mathrm{~cm}$ in length and 1 or $2 \mathrm{~mm}$ in diameter, had a cytoplasmic streaming that was supported by actin cables. We could very readily monitor movement in this system by light microscopy, so it seemed like an ideal system." The idea was a success, and their work showed for the first time clear movement of myosin along actin filaments in an ATP-dependent manner.

Spudich's laboratory was still focused, however, on developing an in vitro system using purified actin rather than relying on the complex Nitella-derived actin substratum. They returned to earlier attempts to reconstitute a totally defined system. In this approach, now involving graduate student Stephen Kron, actin filaments were conjugated to biotin and fixed on avidincoated slides, with buffer flow orienting the actin filaments (Figure 3). Finally, they were able to recreate myosin movement entirely in the test tube, using pure actin, pure myosin, and ATP (7).

\section{A new way of moving}

Just a few floors away from Spudich and Sheetz in the Fairchild Science Building at Stanford, Ronald Vale was studying the development of the nervous system and nerve regeneration. Knowing that axons can reach up to a meter in length, Vale was deeply interested in understanding how long-distance transport occurs between the cell body and terminus of nerve cells. Vale recognized that the work of Spudich and Sheetz had important implications for other kinds of cellular transport. In an interview with the JCI, Vale recalled, "I heard about the exciting result that Mike and Jim got in reconstituting motility in vitro. The images and movies they had looked like, perhaps, how axonal transport might work. The three of us began talking and that seemed like an exciting possibility." In addition to the work of Sheetz and Spudich on myosin, reports by Robert Allen and Scott Brady demonstrated abundant transport of organelles and vesicles within axons, using an approach that combined video with microscopy to monitor giant squid axons $(8,9)$. Building upon these studies, Vale and Sheetz began a serious collaboration to understand the molecular motors driving motility in squid axons.

Sheetz and Vale went to the Marine Biological Laboratory at Woods Hole, where they had ready access to giant squid. Using a cellular extrusion from axons known as axoplasm, they teamed up with electron microscopists, Bruce Schnapp and Thomas Reese, to monitor motility. Initially, Vale and Sheetz thought that nonmuscle myosins might also drive motion in axons. However, they were surprised to find that the molecular motor driving motion in squid axoplasm appeared quite different from myosin. When Sheetz and Vale tested whether myosin conjugated to beads could move in squid axoplasm, they saw no movement. "Since the initial idea wasn't working, we decided to take a more naive approach and really dissect the system step by step to figure out what elements of the cytoskeleton were working. Maybe it wasn't actin, and indeed it turned out microtubules were the main track," recalled Vale.

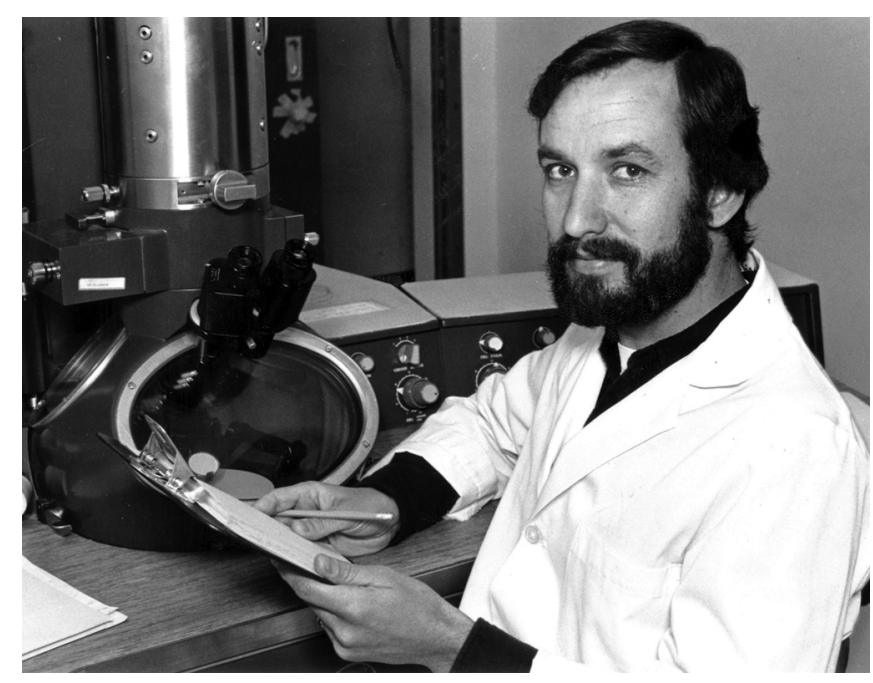

Figure 3

James Spudich at the electron microscope, circa 1984. 


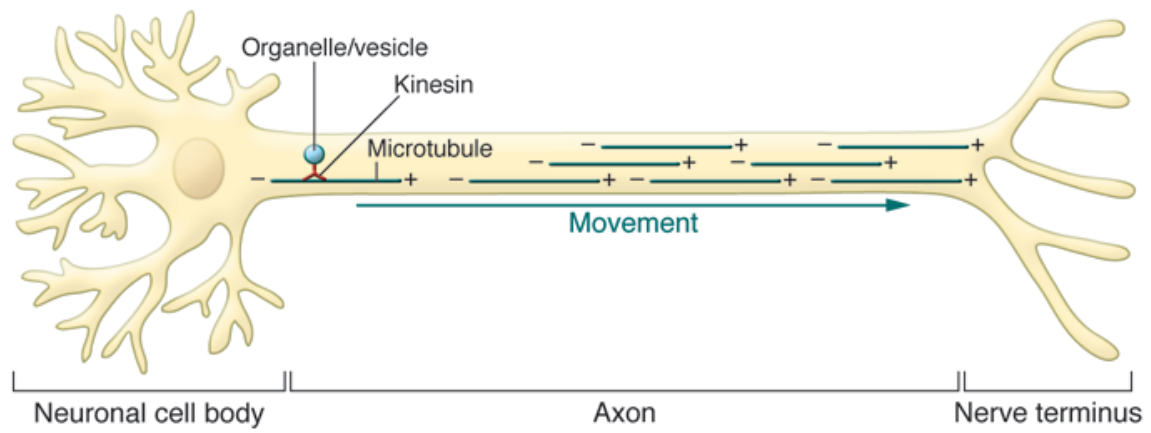

Figure 4

Kinesins generate anterograde movement in cells. Fueled by an interest in how nerves conduct long-distance transport, Ronald Vale and Michael Sheetz examined force-generating proteins in extracts from squid axons. They found that movement occurred along microtubule tracks and discovered kinesin as the molecular motor that drives movement from the cell body to the periphery (10). Another motor protein, dynein (not shown), is responsible for retrograde transport in the opposite direction $(11,12)$.

Once they figured out that microtubules were required for movement, they could test different components and extracts to decipher how movement was generated. Vale and Sheetz soon realized that this motor, unlike myosin, was present in cytoplasmic extracts, and, fortuitously, the motor adhered to glass slides. Sheetz told the JCI, "The breakthrough came when we noticed that microtubules were moving on the glass surface without any vesicles around; there was just supernatant protein catalyzing the movement. It meant that something was different there. And when the molecular weight came out, it was totally different from myosin." Vale and Sheetz had discovered kinesin (Figure 4 and ref. 10). Subsequent work firmly established kinesin as belonging to a new class of motor protein that is not only present in axons, but is fundamental for movement of organelles and vesicles in cells as well as chromosome segregation in cell division. Vale and Sheetz noted that kinesins only generate movement in one direction, away from the center of the cell and toward the periphery, but that another component promotes movement along microtubules in the opposing direction (11). Work by Richard Vallee and colleagues determined that another motor protein, dynein, was responsible for movement along microtubules in the opposite direction (12). Kinesins also promote motility in an ATP-dependent manner, but Vale and graduate student Laura Romberg showed that they differ somewhat from dyneins and myosins in that ATP promotes binding with the microtubule track rather than release (13).

\section{A common mechanism underlying motor activity}

Though myosin family proteins and kinesin family proteins work in distinct ways, subsequent research has unveiled many commonalities in the mechanisms of motor protein function. Actin and microtubules are both comprised of oriented subunits that only allow motor movement in one direction. Kinesins move from the minus end of microtubules to the plus end of microtubules, a polarity that is dictated by the repeating $\alpha$-tubulin and $\beta$-tubulin subunits that make up microtubules. In a manner similar to that of microtubule polarity, asymmetrical actin monomers are assembled into filaments with a directionality that promotes unidirectional movement of myosin. Thus, while kinesin and myosin operate on different tracks, in both cases, the composition of the filament determines the direction of motion.

The motors themselves also turn out to have very similar catalytic engines driving their movement. Spudich, Yoko Yano Toyoshima, and colleagues identified the region in myosin responsible for inducing the sliding movement of actin, the S1 head (14). X-ray crystallography studies of this subfragment (15) subsequently revealed the precise structure of the actin- and nucleotide-binding sites. Soon afterward, Vale and colleagues examined the ATPase domain of kinesin, which showed that the two catalytic domains are structurally very similar, despite little primary sequence homology (16). Though myosin and kinesin have many divergent features, the molecular motors essentially follow the same basic principles to generate movement.

The work of James Spudich, Michael Sheetz, and Ronald Vale provided the fundamental tools to study molecular movement in vitro. Their work was among the first to use light microscopy for biochemistry, allowing them to finely probe the molecular details of how molecular motors function. Sheetz, Vale, and Spudich also reduced the in vitro motility assays to the single molecule level (17-19). The development of optical tweezers for single molecule analysis allowed the measurement of nanometer steps and piconewton forces produced by both kinesin $(17,20)$ and myosin (19). Further, their studies enabled a wealth of subsequent research on the diverse function of the kinesin and myo$\sin$ families in vivo. While the catalysis that drives movement for the 17 classes of myosin proteins and the 14 classes of kinesin family members is essentially the same, the diversity of cellular processes that rely on directed transport is immense. Reflecting on the field, Vale was impressed with the multitude of roles that molecular motors play in the cell, noting that motors are involved in "transporting virtually any kind of material, protein complexes, mRNAs, the nucleus, all the organelles in the cell. Basically, molecular motors are involved in all of the details of where the internal contents of the cell are placed." This key role in spatial organization of cells underlies the importance of molecular motors in muscle contraction, cell division, cell polarity, signal transduction, and many other processes.

Moving forward, the three scientists have engaged in diverse research efforts. Sheetz, now jointly appointed at Columbia University and the National University of Singapore, intensely studies how molecular motors function in sarcomere-like units within nonmuscle cells to sense their local environment. At the University of California, San Francisco, the Vale laboratory is interested in a number of aspects of kinesin biology, including mitosis and $\mathrm{T}$ cell signal transduction. James Spudich and Ronald Vale, along with Lawrence Goldstein and James Sabry, cofounded a company called Cytokinetics that currently has two myosin-activating compounds in clinical trials: Tirasemtiv, for the treatment of amyotrophic lateral sclerosis, and Omecamtiv mecarbil, as a therapeutic for heart failure. Indeed, for Spudich the opportunity to apply findings to the clinic has been incredibly rewarding. "Never in the early 
days did I imagine I would be working on translational research," Spudich said. His laboratory is now entirely devoted to studying the effects of mutations in human cardiac myosins in hypertrophic and dilated cardiomyopathy. Other translational studies in the field have focused on the role of kinesins in cell division and cancer, and targeted therapeutics are under development.

The current advances in the field of molecular motors are, in large part, built upon the seminal findings of Sheetz, Spudich, and Vale. Their successful collaborations led to amazing insight into the basis of molecular motor function and established the three scientists as long-time friends. "All of us have had a long history together," said Vale. The three men were honored and delighted to share in the 2012 Albert Lasker Basic Medical Research Award. Vale noted, "In this case, the prize is shared not just with colleagues in the same field, but with really good friends.”

\section{Sarah Jackson}

1. Huxley AF, Niedergerke R. Structural changes in muscle during contraction: interference microscopy of living muscle fibres. Nature. 1954; 173(4412):147-149.

2. Huxley HE, Hanson J. Changes in the cross striations of muscle during contraction and stretch and their structural interpretation. Nature. 1954; 173(4412):973-976.

3. Huxley HE. The mechanism of muscular contraction. Science. 1969;164(3386):1356-1365.

4. Clarke M, Spudich JA. Biochemical and structural studies of actomyosin-like proteins from non-muscle cells. Isolation and characterization of myosin from amoebae of Dictyostelium discoideum. J Mol Biol. 1974;86(2):209-222.

5. De Lozanne A, Spudich JA. Disruption of the Dictyostelium myosin heavy chain gene by homologous recombination. Science. 1987;236(4805):1086-1091.

6. Sheetz MP, Spudich JA. Movement of myosincoated fluorescent beads on actin cables in vitro. Nature. 1983;303(5912):31-35.

7. Spudich JA, Kron SJ, Sheetz MP. Movement of myosin-coated beads on oriented filaments reconstituted from purified actin. Nature. 1985;315(6020):584-586.

8. Allen RD, Metuzals J, Tasaki I, Brady ST, Gilbert SP. Fast axonal transport in squid giant axon. Science. 1982;218(4577):1127-1129.

9. Brady ST, Lasek RJ, Allen RD. Fast axonal transport in extruded axoplasm from squid giant axon. Science. 1982;218(4577):1129-1131.

10. Vale RD, Reese TS, Sheetz MP. Identification of a novel force-generating protein, kinesin, involved in microtubule-based motility. Cell. 1985;42(1):39-50.
11. Vale RD, Schnapp BJ, Mitchison T, Steuer E, Reese TS, Sheetz MP. Different axoplasmic proteins generate movement in opposite directions along microtubules in vitro. Cell. 1985;43(3):623-632.

12. Paschal BM, Vallee RB. Retrograde transport by the microtubule-associated protein MAP 1C. Nature. 1987;330(6144):181-183.

13. Romberg L, Vale RD. Chemomechanical cycle of kinesin differs from that of myosin. Nature. 1993;361(6408):168-170.

14. Toyoshima YY, Kron SJ, McNally EM, Niebling KR, Toyoshima C, Spudich JA. Myosin subfragment-1 is sufficient to move actin filaments in vitro. Nature. 1987;328(6130):536-539.

15. Rayment I, et al. Three-dimensional structure of myosin subfragment-1: a molecular motor. Science. 1993;261(5117):50-58.

16. Kull FJ, Sablin EP, Lau R, Fletterick RJ, Vale RD. Crystal structure of the kinesin motor domain reveals a structural similarity to myosin. Nature. 1996;380(6574):550-555.

17. Gelles J, Schnapp BJ, Sheetz MP. Tracking kinesindriven movements with nanometre-scale precision. Nature. 1988;331(6155):450-453.

18. Howard J, Hudspeth AJ, Vale RD. Movement of microtubules by single kinesin molecules. Nature. 1989;342(6246):154-158.

19. Finer JT, Simmons RM, Spudich JA. Single myosin molecule mechanics: piconewton forces and nanometre steps. Nature. 1994;368(6467):113-119.

20. Svoboda K, Schmidt CF, Schnapp BJ, Block SM. Direct observation of kinesin stepping by optical trapping interferometry. Nature. 1993;365(6448):721-727. 